\title{
Relación entre Estrategias Competitivas y Tipos de Aprendizaje Organizativo en Empresas Colombianas
}

\author{
Esteban López-Zapata, Gloria P. López-Moros y Sabrina M. Agudelo-Muñoz \\ Universidad de Antioquia, Facultad de Ciencias Económicas. Calle 70 N52-21, Oficina 13-403. Medellín, Colombia \\ (e-mail: esteban.lopez@udea.edu.co; glorypati@gmail.com; sabrinagudelo01@gmail.com)
}

Recibido Dic. 17, 2018; Aceptado Feb. 21, 2019; Versión final Abr. 12, 2019, Publicado Oct. 2019

\begin{abstract}
Resumen
El objetivo de este trabajo es analizar la relación existente entre las estrategias competitivas de ventaja en costos, diferenciación de producto y estrategia hibrida con tres tipos de aprendizaje organizativo: explotación, exploración y ambidestreza. Para ello se aplicó un cuestionario a una muestra de 313 directivos de empresas colombianas, se utilizó la metodología de análisis factorial confirmatorio para validar las escalas y análisis de regresión múltiple para contrastar las hipótesis. Se comprobó que existe una relación positiva entre la estrategia de ventaja en costos con el aprendizaje de explotación, la estrategia de diferenciación de producto con el aprendizaje de exploración y la estrategia híbrida con el aprendizaje ambidiestro. Se concluye que cada tipo de estrategia competitiva se ajusta de forma particular con cada tipo de aprendizaje organizativo.
\end{abstract}

Palabras clave: estrategia competitiva; aprendizaje organizativo; exploración; explotación; ambidestreza

\section{Relationship between Competitive Strategies and Types of Organizational Learning in Colombian Companies}

\begin{abstract}
The objective of this paper is to analyze the relationship between competitive strategies of cost advantage, product differentiation and hybrid strategy with three types of organizational learning: exploitation, exploration and ambidexterity. To this end, a questionnaire was applied to a sample of 313 managers of Colombian companies. Confirmatory factor analysis was used to validate the scales and multiple regression analysis to test the hypotheses. It was found that there is a positive relationship between the cost advantage strategy with exploitation learning, the product differentiation strategy with exploration learning and the hybrid strategy with ambidextrous learning. It is concluded that each type of competitive strategy is adjusted in a particular way with each type of organizational learning.
\end{abstract}

Keywords: competitive strategy; organizational learning; exploration; exploitation; ambidexterity 


\section{INTRODUCCIÓN}

Desde hace varios años, el aprendizaje organizativo viene despertando el interés de académicos y profesionales de la administración. Por esta razón, el aprendizaje viene siendo abordado como un activo empresarial, no solo ligado a la inteligencia de un individuo, sino también a los equipos de trabajo y las organizaciones (Crossan et al., 1999; Easterby-Smith et al., 2000). A pesar de los avances en materia de aprendizaje organizativo, aún es incipiente el desarrollo y profundización en el aprendizaje de exploración, explotación y ambidiestro, sin embargo, en los últimos años ha crecido el interés por comprender el rol que cada uno de ellos desempeña en las organizaciones y las actividades que los conforman.Para el caso de la exploración y la explotación, ambas habilidades tienen dos enfoques diferentes en el aprendizaje organizativo, pero la literatura en las últimas décadas ha expuesto la necesidad en las organizaciones de lograr un equilibrio entre las dos (He y Wong, 2004). Ese equilibrio está inmerso en el concepto de aprendizaje ambidiestro, que hace referencia al desarrollo combinado de las capacidades de explotación y exploración, es decir, una organización ambidiestra tiene la habilidad de explotar sus conocimientos acumulados, pero también está abierta a explorar nuevas bases de conocimientos; es un tipo de capacidad que permite a las empresas rentabilizar su saber y mejorar su eficiencia de forma gradual en sus negocios actuales, al mismo tiempo que se prepara para innovar, adaptarse al cambio y enfrentar mejor sus negocios futuros (Raisch y Birkinshaw, 2008; Van Grinsven y Visser, 2011). El aprendizaje organizativo, por sí solo no apoya el éxito empresarial, debe estar acompañado de otros factores como los recursos, la dirección, la planeación y la estrategia, aspectos que se engranan para el cumplimiento de los objetivos empresariales y que finalmente pretenden asegurar la competitividad, capacidad clave que determina los factores diferenciadores y posibilita el valor agregado para los grupos de interés de una organización.

Los estudios existentes sobre el aprendizaje ambidiestro están en su mayoría relacionados con variables como liderazgo, gestión humana y desempeño (Turner et al., 2013; Junni et al., 2015), sin embargo hay poca evidencia directa sobre su relación con la estrategia competitiva, asunto que es importante, ya que los mercados son dinámicos y por tanto las organizaciones y sus directivos deben estar preparados para atender todos los frentes e interrogantes empresariales y dar en general un manejo táctico y discrecional, que facilite el logro de resultados exitosos, redundantes en el fortalecimiento competitivo y de proyección a largo plazo. Partiendo de la necesidad de estudiar una relación poco ahondada y de la revisión bibliográfica sobre las estrategias competitivas de liderazgo en costos, diferenciación y estrategia híbrida, como también del aprendizaje ambidiestro, incluidas sus actividades de exploración y explotación; este artículo tiene por objeto evaluar el impacto de la estrategia de diferenciación sobre el aprendizaje de exploración, el liderazgo en costos sobre el aprendizaje de explotación y de la estrategia híbrida sobre el aprendizaje ambidiestro. Por otra parte, se pretende difundir un tema contemporáneo y poco estudiado en el contexto colombiano, que concede un aporte interesante a los modelos de administración existentes, principalmente a los directivos que propenden por un negocio próspero y perseverante en el tiempo.

Para esto, se aplicó un cuestionario a una muestra de 313 directivos de empresas colombianas y a través de un análisis estadístico de modelos de regresión lineal, se encontró evidencia de que la estrategia de diferenciación de productos tiene una relación positiva con el aprendizaje de exploración, la estrategia de liderazgo en costos una relación positiva con el aprendizaje de explotación y la estrategia híbrida una relación positiva con el aprendizaje ambidiestro. Finalmente se presenta la discusión, conclusiones y posibles líneas de investigación que pueden impulsar la evolución del tema de la ambidestreza organizativa y motivar la profundización de su relación con la estrategia competitiva.

\section{ESTRATEGIA COMPETITIVA Y APRENDIZAJE ORGANIZATIVO}

La estrategia competitiva puede definirse como la fórmula que implementa una empresa para alcanzar un rendimiento superior al de sus competidores. Es un método que encamina una serie de actividades, para crear una posición tolerable a un entorno ofensivo, y que promueve la capacidad para emprender acciones defensivas en el sector en el cual se desempeñe una organización, facilitando así un rendimiento superior sobre su competencia (Porter, 1985). El estudio de estos factores y la estrategia implementada, le dan forma a la competencia del sector y posibilitan la identificación de la posición relativa de la empresa dentro de su industria, facilitando así el logro de una ventaja competitiva sostenida, que redunda en una rentabilidad mayor, por encima a la media de su sector económico, porque finalmente estos procesos se reducen a un objetivo general, la generación de valor para los socios o accionistas (Navas y Guerras, 2012). Las estrategias competitivas de Porter (1980) se definieron a partir de la relación entre el tipo de ventaja que se desea alcanzar con el entorno para el cual se pretende conseguir dicha ventaja, estas son: liderazgo en costos, diferenciación y enfoque en un nicho de mercado. Esta investigación se centra en las estrategias dirigidas al ámbito de la industria, es decir, en costes, en diferenciación y la combinación de ambas, conocida como estrategia híbrida. 
Desde el enfoque basado en los recursos (Barney, 1991), la elección de la estrategia depende de la dotación de recursos que posee la empresa y el potencial que tienen dichos recursos para apalancar la ventaja competitiva, es decir, cuando dichos recursos son valiosos, escasos, difíciles de imitar y difíciles de sustituir. El aprendizaje organizativo puede ser un mecanismo para desarrollar recursos de gran potencial y también puede ser una capacidad dinámica (Teece, 2007) ya que permite construir competencias internas y externas, permitiendo explotar los recursos actuales e identificar oportunidades de innovación, convirtiéndose así en una de las herramientas más poderosas de las organizaciones para crear una ventaja competitiva sostenida, siendo difícil de comprender, de imitar o de interiorizar por los competidores (Barney, 1991). La capacidad de aprendizaje y gestión del conocimiento se ha identificado como un factor crítico que ayuda a explicar la creatividad organizacional (Echeverri et al., 2018) y la competitividad (Simanca et al., 2016).

Existen dos dimensiones del aprendizaje organizativo: una es la exploración, que corresponde a la búsqueda, asunción de riesgos, flexibilidad, experimentación, descubrimiento e innovación, y la otra es la explotación que se concentra en la producción actual, la eficiencia, el refinamiento, la implementación y ejecución (March, 1991). Cada una de ellas se relaciona con rutinas administrativas y conductas gerenciales diferentes. La explotación implica un proceso en el que altos directivos actúan y gestionan para institucionalizar hábitos y comportamientos más adecuados, con el objetivo de perfeccionar las competencias actuales, mientras la exploración implica un proceso donde los altos directivos deben convencerse de reemplazar sus antiguas costumbres y desarrollar nuevos planes de acción, que contribuyen a cambios positivos para la organización (Lubatkin et al., 2006).En años recientes ha surgido un enfoque teórico de la organización ambidiestra, que ha centrado sus esfuerzos en conciliar las dimensiones antes mencionadas (explotación y exploración) ya que ambos representan nortes aparentemente contradictorios: aprovechar los conocimientos actuales para ser eficientes en sus actuales negocios y adquirir nuevos conocimientos que le permitan construir nuevas oportunidades (Raisch y Birkinshaw, 2008). La diferencia existente entre las características de las actividades que contemplan la exploración y la explotación ha generado un dilema teórico entre diferentes autores, ya que para algunos estas capacidades no pueden ejecutarse simultáneamente en una organización, porque cada una está enmarcada en naturalezas completamente diferentes. Luego, exponen que deben ser planteadas por separado creando unidades independientes (March, 1991; Boumgarden et al., 2012).

No obstante, hay estudios que apoyan el modelo de ambidestreza organizativa y proponen el desarrollo de mecanismos, que le permitan a la organización el planteamiento de las actividades de explotación y exploración en una misma unidad de forma paralela (Raisch y Birkinshaw, 2008). Como lo argumentan Baum, et al. (2000) y Gupta et al. (2006), cuando una empresa tiene la capacidad de aprender a partir de su propia experiencia, está logrando un aprendizaje de explotación y cuando a su vez es capaz de aprender a partir de la experiencia de los otros, está logrando un aprendizaje de exploración, justificando así que son dos variables independientes pero que no necesariamente tienen que ser excluyentes. Así, la capacidad de las empresas, de ser diestras simultáneamente en las actividades que relacionan la explotación y la exploración es lo que se define como la ambidestreza organizativa (López-Zapata et al., 2012). Aunque cada vez cobra más relevancia el estudio del aprendizaje ambidiestro y las diferencias entre las actividades que representan la exploración y explotación, aún son escasas las investigaciones que relacionan estos elementos con las estrategias competitivas. El núcleo de investigaciones en este campo comúnmente está direccionado al diseño, liderazgo y desempeño de las organizaciones. De acuerdo con esto, se considera importante desarrollar el concepto de estrategias competitivas, su relación con la exploración y explotación, y posteriormente verificar el impacto que puedan tener sobre las mismas.

La estrategia de diferenciación, hace referencia a los atributos de los productos o servicios, que son percibidos como únicos por parte del mercado (Porter, 1980). Los departamentos de Investigación, desarrollo e innovación $(\mathrm{I}+\mathrm{D}+\mathrm{i})$ y de marketing cumplen un papel fundamental en esta estrategia y más en la época actual, en la que el cliente tiene un alto grado de exigencia y preferencia por lo novedoso, exclusivo y sobre todo si le brinda estatus. La diferenciación no sólo se le atribuye al producto o servicio, hay otras fuentes de aplicación como son los procesos de fabricación, tecnologías empleadas, el conocimiento patentado, el marketing, amplitud de la línea de productos, alcance geográfico, servicio postventa, entre otros. Uno de los mayores retos que busca esta estrategia, es lograr un diseño e imagen aceptable en el cliente, de tal manera que lo fidelice con la empresa y esté dispuesto a sacrificar el pago de un mayor precio a cambio de una retribución en mayor comodidad, tranquilidad, seguridad y singularidad (Porter, 1985). Este enfoque va de la mano con el concepto de empresas prospectivas o buscadoras, de acuerdo con la clasificación que hicieron Miles y Snow (1978), respecto a los tipos de comportamiento que adoptan las organizaciones para seguir estrategias que les permita permanecer en un medio en constante evolución, este es el tipo de empresa que se anticipa y busca ser pionera por medio de la inversión en investigación, desarrollo e innovación ( $1+\mathrm{D}+\mathrm{i})$. El aprendizaje de exploración es el conocimiento que tiene como base la búsqueda, la experimentación, flexibilidad, descubrimiento e innovación, para una empresa (March, 1991). Es el aprendizaje que permite a la empresa evolucionar y adaptarse a los cambios del entorno (López-Zapata et al., 2012). 
Los conceptos de diferenciación de productos y aprendizaje de exploración tienen un componente en común relevante, que hace las veces de impulsor en cada uno y es la innovación, precisamente porque la necesidad de permanencia y continuidad de una empresa exige cambios importantes, que aporten distinción, recordación y reconocimiento, por lo que se encuentra una relación directa entre estos dos conceptos, y por esta razón se infiere que el impacto de la estrategia de diferenciación de producto sobre el aprendizaje de exploración es positivo. Por tanto se presenta la primera hipótesis: H1: La estrategia de diferenciación de productos tiene un impacto positivo sobre el aprendizaje de exploración de una organización.

Por otro lado, la estrategia de ventaja en costos es la segunda de las estrategias competitivas básicas, conforme con la clasificación que realizó Porter (1980). Esta estrategia se centra en la realización de operaciones productivas y empresariales de una forma distinta, propia, focalizada en el manejo eficiente de los costos, sin descuidar la calidad. La estrategia de ventaja en costos implica que la empresa realiza una enérgica búsqueda de reducción de los costos a partir de la curva de experiencia, como consecuencia del aprendizaje. La curva de aprendizaje consiste en la disminución del tiempo en la elaboración de una unidad de producto o prestación de un servicio, a medida que el proceso se repita mayor número de veces, logrando así que el coste total de un producto o servicio disminuya, conforme aumenta la producción acumulada (Yelle, 1979). Otros factores que favorecen la disminución de costos son las mejoras en los procesos, el rediseño de productos o servicios y las economías de escala. Las fuentes de ventaja en costos están en muchas áreas de la organización, generalmente en las que tienen una parte importante de costos fijos, como son publicidad, fuerza de ventas, servicios administrativos e I+D, asimismo en áreas que manejan costos variables como producción, compras y distribución (Porter, 1985; Bowman y Faulkner, 1997). Lo esencial es tratar de mantener bajos costes en comparación con los competidores, sin descuidar asuntos de calidad, servicio, ni otros aspectos. Estratégicamente, la empresa que desea obtener una ventaja en costos debe identificar oportunidades internas en áreas en las cuales pueda desarrollar mayores economías de escala que sus competidores (Bowman y Faulkner, 1997), para lo cual puede ser fundamental el aprendizaje de explotación, que permitiría obtener un máximo provecho de la eficiencia potencial de la organización. Este enfoque conforme a la clasificación de Miles y Snow (1978), se puede asociar al concepto de empresas defensoras, que básicamente son muy conservadoras y controladoras de su nicho de mercado, prefieren la estabilidad a la investigación o exploración de oportunidades, lo que se traduce en el manejo cuidadoso de recursos y centran su interés en incrementar la eficiencia de sus operaciones actuales.

El aprendizaje de explotación se refiere al conocimiento que ya posee la organización, se incorpora con términos como implementación, eficiencia, producción, refinamiento (March, 1991). Es el aprendizaje que permite rentabilizar el conocimiento existente en la organización y así mismo mejorar la eficiencia de forma gradual (López-Zapata et al., 2012). El punto en el que se relacionan la estrategia de ventaja en costos, con el aprendizaje de explotación es la eficiencia en el manejo de sus recursos, de un lado los costos y por el otro el conocimiento, pero moviéndose en el eje de lo existente, de lo que se posee actualmente. La explotación de la base de conocimientos que la organización ya posee se refleja en la acumulación de experiencia y en la curva de aprendizaje de las tecnologías actuales de la empresa, la cual es precisamente una de las principales fuentes de la ventaja en costos. Por esta razón la estrategia de liderazgo en costos presenta una relación con el aprendizaje de explotación, en la medida que se logre optimizar. Es decir, la implementación de esta estrategia exigirá mayor productividad y rentabilidad con el mismo nivel de recursos, y por ende se requerirá una explotación de conocimiento mayor, que redunde en buenas prácticas administrativas, maximización de la producción acumulada y el aumento de la cuota de mercado. Por lo anterior, se presenta la segunda hipótesis: H2: La estrategia de liderazgo en costos tiene un impacto positivo sobre el aprendizaje de explotación de una organización.

A pesar de la amplia definición de Porter (1985) sobre los tipos de estrategias genéricas y el análisis de las bondades de cada una de estas orientaciones, el autor empleó la expresión "atrapada en la mitad", para referirse a las empresas que no se centran en una de estas direcciones (bajo costo o diferenciación), según él, ambas estrategias son incompatibles para lograr una ventaja competitiva y superar a otras empresas de la misma industria, porque cada una demanda unos recursos, habilidades y organización diferente. La falta de concentración en una sola estrategia conlleva a no alcanzar ninguna, es una manifestación de falta de claridad para tomar decisiones acerca de cómo competir, baja proyección a un alto mercado, además de una subordinación entre estas, porque el logro de una mayor diferenciación se traduce en mayores costos (Pertusa-Ortega et al., 2009). Por ende, las organizaciones quedan en una situación muy difícil para competir, porque su cuota de mercado es baja, presentan dificultades con su inversión de capital, pérdida de rentabilidad y tienen prácticamente una sentencia al fracaso. Es una combinación estratégica sin éxito. De acuerdo a Porter (1980), la elección entre estas opciones se debe fundamentar en las capacidades y limitaciones de la empresa, porque la práctica de cada estrategia comprende un estilo de dirección, pautas de organización, diferentes recursos. El dilema se puede resolver, al escoger la estrategia que mejor se adapte a las fortalezas de la empresa, que sea más difícil de imitar por los competidores (Porter, 1985; PertusaOrtega et al., 2009). 
Sin embargo, diversos autores plantean que las organizaciones son aptas para manejar más de una estrategia genérica a la vez sin quedar necesariamente "atrapada a la mitad". Por ejemplo, las denominadas estrategias de océano azul se caracterizan por buscar simultáneamente la diferenciación y bajo costo a través de la innovación en valor, lo cual se logra cuando los actos de la empresa inciden favorablemente sobre su estructura de costos y sobre la propuesta de valor para los compradores, reduciendo variables sobre las cuales se compite tradicionalmente en la industria y creando atributos que la industria nunca ha ofrecido a los clientes (Kim y Mauborgne, 2005).Surge entonces el concepto de estrategia híbrida, que se refiere básicamente a la combinación de las estrategias de ventaja en costos y diferenciación. Pertusa-Ortega et al. (2009) aseguran que a este tipo de estrategia se le ha dedicado menos atención en las investigaciones y más aún a su relación con el desempeño empresarial, en cambio, se han focalizado más en determinar si la estrategia de las empresas puede estar representada en uno de los tipos genéricos propuestos por Porter (1980) y la compatibilidad o no entre las mismas, de las cuales se han obtenido conclusiones que en general determinan que estas estrategias son excluyentes entre sí porque, por ejemplo, para lograr grandes diferenciaciones se afectan los costos y se actúa con menos estandarización de procesos.

No obstante, estos autores se apoyaron en dos argumentos, para defender la factibilidad de estas estrategias conjuntamente, el primero se refiere a que ser fuerte en una estrategia competitiva, puede impulsar la mejora de la otra. Una sólida posición en diferenciación provoca un aumento de la demanda, por ende un aumento de la cuota de mercado, facilitando así una explotación de las economías de escala, que básicamente trascienden en una mejora de los indicadores de eficiencia. Por otra parte, una firme postura en costos, facilita la inversión de utilidades directamente en los atributos de servicios o productos, reforzando la estrategia de diferenciación (Pertusa-Ortega et al., 2009). El segundo argumento, hace referencia a ciertos hábitos empresariales que impulsan el posicionamiento de ambas estrategias, como son: la gestión de calidad y la gestión ambiental, procedimientos que implícitamente tienen unas actividades orientadas a cumplir con la propuesta de valor hacia el cliente y un enfoque de optimización de los recursos que analizados a fondo, son propiamente la orientación a la estrategia de diferenciación y de liderazgo en costos, que confluyen en la producción de bienes y servicios con buena reputación, y que se traducen en mejores niveles de competitividad, incremento de las ventas, mayores utilidades y un alto nivel de desempeño de la empresa (Pertusa-Ortega et al., 2009). En consecuencia, puede decirse que las empresas que se orientan por una estrategia híbrida, se enfatizan en alcanzar eficiencia (bajos costos, alta diferenciación), son difíciles de imitar por sus competidores, dedican atención a varios asuntos de mercadeo para satisfacer una cuota de mercado significativa a través de mejores atributos de sus productos o servicios, pueden asumir retos para responder a las nuevas tendencias y necesidades de los clientes; mientras que las organizaciones que se encuentran "atrapadas en la mitad" se enmarcan en una baja ventaja competitiva (altos costos y baja diferenciación), son más vulnerables a plagios por parte de la competencia, su concentración en pocos asuntos es negativa, porque desatienden las necesidades variables de los clientes, son inflexibles a los cambios y tiene poca proyección empresarial (Pertusa-Ortega et al., 2009; Baroto et al., 2012). Lo ideal entonces, sería que las empresas pudieran combinar los costos y la diferenciación (estrategia híbrida), de forma equilibrada, como fuente de ventaja competitiva.

En las dos hipótesis anteriores fue posible hallar una relación directa entre las estrategias de diferenciación de productos con el aprendizaje de exploración y la estrategia de ventaja en costos con el aprendizaje de explotación, ahora se considera interesante analizar la relación entre la estrategia híbrida (ventaja en costos y diferenciación), con el aprendizaje ambidiestro (exploración y explotación). En el mundo, varias compañías vienen implementando esta estrategia con éxito, porque han desarrollado la capacidad de ofrecer mayor valor añadido a los clientes en los productos o servicios con precios más bajos, y sin afectar los márgenes de rentabilidad en la medida que se logran mantener altas cuotas de mercado. A medida que aumenta la competencia global, se le viene dando mayor importancia a la estrategia híbrida (Baroto et al., 2012), la cual representa un reto grande y exigente para una organización, porque debe asegurar la retención de los consumidores actuales, aumentar la cuota de mercado, consolidar un mayor índice de rentabilidad y a la vez conservar un nivel de costes relativamente bajos, sin afectar la calidad. La estrategia híbrida y el aprendizaje ambidiestro lógicamente tienen conceptos e implicaciones distintos, la hibridación de ventaja en costos y diferenciación se ocupa básicamente de aspectos externos, de cara al cliente y de la competencia. Mientras que la ambidestreza organizativa se ocupa del aprendizaje y capacidades dinámicas organizativas, con alto interés en la optimización de las habilidades adquiridas y el cambio de rutinas, para determinar una identidad corporativa. De otro lado, ambos conceptos son complementarios, la estrategia híbrida se encarga de definir la propuesta de valor de una empresa y la ambidestreza se centra en la forma de entregar ese valor con eficiencia (explotación) y de cómo renovar el conocimiento de manera eficaz (exploración). Aunque son dos enfoques distintos, buscan gestionar objetivos empresariales con efectos complementarios a corto plazo, para la sostenibilidad a largo plazo, asimismo, comparten el propósito de desarrollar una capacidad de adaptación al medio ambiente complejo de una industria (Lapersonne et al., 2015). A partir de esta situación, que se ve representada en dos dimensiones diferentes pero complementarias, se plantea la tercera hipótesis: $H 3$ : $L a$ estrategia híbrida tiene un impacto positivo sobre el aprendizaje ambidiestro. 


\section{METODOLOGÍA}

Se describen los procesos utilizados para seleccionar la muestra de la investigación y las escalas de medida utilizadas para contrastar las hipótesis. Se presentan también los procedimientos utilizados para evaluar la fiabilidad y validez de dichas escalas.

\section{Población y muestra}

Para evaluar las hipótesis planteadas, se recopilaron los datos por medio de un cuestionario virtual aplicado a directivos de grandes, medianas y pequeñas empresas colombianas. Utilizando la base de datos Gestor Comercial, que está conformada por más de 45.000 empresas de todas las ciudades de Colombia, se obtuvo una muestra de 313 directivos de organizaciones del sector manufacturero y de servicios (lo cual corresponde a un margen de error del 5,5\% con un nivel de confianza del 95\%). La encuesta se remitió a través de correo electrónico a los gerentes, directores y administradores generales de las empresas. Se realizó una prueba piloto del cuestionario a 22 empresas que se ajustaron al perfil, para verificar que las afirmaciones fueran claras, relevantes y se interpretaran de acuerdo a lo esperado. La muestra fue clasificada de acuerdo a tres variables: tamaño, sector y edad de las empresas. En la tabla 1 se presenta el resumen de clasificación de la muestra de acuerdo a estas tres variables.

Tabla 1: Variables de clasificación de la muestra

\begin{tabular}{|l|l|l|l|}
\hline Variable de clasificación & Categorías & Frecuencia & Porcentaje \\
\hline \multirow{4}{*}{ Tamaño } & Grande & 145 & 46,3 \\
& Mediana & 62 & 19,8 \\
& Pequeña & 106 & 33,9 \\
& Total & 313 & 100,0 \\
\hline \multirow{2}{*}{ Sector } & Manufactura & 74 & 23,6 \\
& Servicios & 239 & 76,4 \\
& Total & 313 & 100,0 \\
\hline \multirow{5}{*}{ Edad } & 0 a 20 & 137 & $43,8 \%$ \\
& 21 a 40 & 78 & $24,9 \%$ \\
& 41 a 60 & 60 & $19,2 \%$ \\
& 61 a 100 & 38 & $12,1 \%$ \\
\hline & Total & 313 & $100,0 \%$ \\
\hline
\end{tabular}

\section{Medidas}

En la literatura revisada se identificaron algunos autores que han utilizaron escalas de medidas para los tipos de aprendizaje de exploración, explotación y ambidiestro, así como aquellos que han utilizado escalas para medir las estrategias competitivas de ventaja en costos, diferenciación e híbrida. Todo el instrumento se desarrolló utilizando escalas de Likert de 5 puntos (1: Totalmente en desacuerdo, 5: Totalmente de acuerdo). Para la medición de los constructos de aprendizaje organizativo se utilizaron tres ítems para medir el aprendizaje de exploración y tres ítems para medir el aprendizaje de explotación, los cuales se desprenden de las escalas desarrolladas por Lubatkin et al. (2006). Para medir las ambidestreza organizativa se utilizó la interacción (multiplicación) entre las escalas de exploración y explotación, tal como lo proponen He y Wong (2004). Para los constructos de las estrategias competitivas se usaron tres ítems para medir la estrategia de ventaja en costos y tres para medir la estrategia de diferenciación de producto, la elaboración de estos se hizo con base en las escalas de Bowman (1991) y de Claver-Cortés et al. (2012). Para medir la estrategia híbrida se utilizó la interacción (multiplicación) entre las escalas de costos y diferenciación. Esta investigación empleó las siguientes variables de control, de acuerdo con las características de las empresas encuestadas: sector (manufacturero y servicios), tamaño (grande, mediana y pequeña) y edad (años de existencia).

Para la verificación de fiabilidad y validez del instrumento construido se procedió a la realización de un Análisis Factorial Confirmatorio utilizando el software EQS, con el método de máxima verosimilitud robusto. En la tabla 2 , se presentan cada uno de los constructos con las variables empleadas para el cuestionario y los principales resultados del Análisis Factorial Confirmatorio. Para asegurar la validez convergente de las escalas se evaluó que todos los ítems tuvieran cargas factoriales significativas superiores a 0,6 y que el promedio de dichas cargas factoriales fuera superior a 0,7 para cada constructo (Hair et al., 1999). De igual forma se encontró que el modelo presentaba una bondad de ajuste adecuada. El valor de Chi-cuadrado de Satorra-Bentler fue de 273,1463 (g.l. = 109; p<0,01). Sin embargo, teniendo en cuenta el tamaño de la muestra (superior a 200 observaciones) resulta más pertinente analizar los índices de bondad de ajuste que el valor directo de la Chicuadrado (Hair et al., 1999). Los índices BBNFI, BBNNFI, CFI e IFI presentaron todos valores superiores a $0,9$ (BBNFI=0,905; $\mathrm{BBNNFI}=0,925 ; \mathrm{CFI}=0,940 ; \mathrm{IFI}=0,941)$ y el error de aproximación cuadrático RMSA es inferior a 0,08 (RMSEA $=0,069[0,059-0,080])$. 
Tabla 2: Constructos, variables y análisis de validez convergente

\begin{tabular}{|c|c|c|c|c|c|}
\hline Constructo & Código & $\begin{array}{c}\text { Cargas } \\
\text { Factoriales } \\
\text { Estandarizadas }\end{array}$ & $\begin{array}{c}\text { Promedio } \\
\text { Cargas } \\
\text { Factoriales } \\
\text { Estandarizadas }\end{array}$ & $R^{2}$ & Variables \\
\hline \multirow{3}{*}{$\begin{array}{l}\text { Aprendizaje de } \\
\text { exploración }\end{array}$} & EXR1 & 0,837 & \multirow{3}{*}{0,820} & 0,70 & $\begin{array}{l}\text { Buscamos nuevas ideas tecnológicas } \\
\text { pensando más allá de lo habitual }\end{array}$ \\
\hline & EXR2 & 0,842 & & 0,71 & $\begin{array}{l}\text { Basamos nuestro éxito en la capacidad de } \\
\text { explorar nuevas tecnologías }\end{array}$ \\
\hline & & 0,781 & & 0,61 & $\begin{array}{l}\text { Creamos productos que son innovadores } \\
\text { en la empresa }\end{array}$ \\
\hline \multirow{3}{*}{$\begin{array}{l}\text { Aprendizaje de } \\
\text { explotación }\end{array}$} & EXT1 & 0,835 & \multirow{3}{*}{0,821} & 0,70 & $\begin{array}{l}\text { Estamos comprometidos en mejorar la } \\
\text { calidad de los productos y bajar los costos }\end{array}$ \\
\hline & EXT2 & 0,899 & & 0,81 & $\begin{array}{l}\text { Mejoramos continuamente la confiabilidad } \\
\text { de nuestros productos y servicios }\end{array}$ \\
\hline & & 0,728 & & 0,53 & $\begin{array}{l}\text { Mejoramos nuestra oferta para mantener a } \\
\text { los clientes actuales satisfechos }\end{array}$ \\
\hline \multirow{3}{*}{$\begin{array}{l}\text { Estrategia de } \\
\text { costos }\end{array}$} & COST1 & 0,767 & \multirow{3}{*}{0,826} & 0,59 & $\begin{array}{l}\text { Enfatizamos considerablemente en el } \\
\text { control de costos operacionales }\end{array}$ \\
\hline & COST2 & 0,869 & & 0,76 & $\begin{array}{l}\text { Nos esforzamos en hacer una utilización } \\
\text { más eficiente de nuestros recursos }\end{array}$ \\
\hline & COST3 & 0,842 & & 0,71 & $\begin{array}{l}\text { Monitoreamos cuidadosamente las } \\
\text { operaciones para ayudarnos a mantener } \\
\text { los costos bajo control }\end{array}$ \\
\hline \multirow{3}{*}{$\begin{array}{l}\text { Estrategia de } \\
\text { diferenciación }\end{array}$} & DIF1 & 0,919 & \multirow{3}{*}{0,790} & 0,85 & $\begin{array}{l}\text { Desarrollamos una promoción intensiva de } \\
\text { nuestra marca }\end{array}$ \\
\hline & DIF2 & 0,691 & & 0,48 & $\begin{array}{l}\text { Invertimos significativamente en nuestra } \\
\text { fuerza de ventas }\end{array}$ \\
\hline & DIF3 & 0,760 & & 0,58 & $\begin{array}{l}\text { Desarrollamos campañas publicitarias para } \\
\text { reforzar nuestra imagen de marca }\end{array}$ \\
\hline
\end{tabular}

El análisis de validez discriminante se presenta en la Tabla 3. Los valores de la diagonal de la matriz corresponden a la raíz cuadrada de la varianza extraída de cada factor. Los valores debajo de la diagonal corresponden a los intervalos de confianza para la correlación entre factores. Los valores sobre la diagonal son las correlaciones estimada entre cada par de factores. Se valoró la validez discriminante verificando que el valor 1 no estuviera en el intervalo de confianza de las correlaciones entre los diferentes constructos (Anderson y Gerbing, 1988) y que la raíz cuadrada del Índice de Varianza Extraída de cada factor fuera superior a la correlación estimada entre cada par de factores (Fornell y Larcker, 1981). Este último criterio sólo se incumple entre el factor 2 y 3 , pero teniendo en cuenta que es una diferencia poco significativa y que se cumple el primer criterio, se considera que las escalas tienen suficiente validez discriminante.

Finalmente, para verificar la fiabilidad, se calculó el Alpha de Cronbach para cada constructo (Tabla 4), y en cada uno de ellos se verificó que fueran mayores a 0,7 (Hair et al., 1999). Adicionalmente, se calculó el Índice de Fiabilidad Compuesta (IFC) y el Índice de Varianza Extraída (IVE), verificando que fueran superiores a 0,7 y 0,5 respectivamente (Fornell y Larcker, 1981). Posterior a la verificación de fiabilidad de las variables en estudio, con los resultados obtenidos de la aplicación del instrumento, se realizaron los modelos de regresión múltiple para contrastar las hipótesis de la investigación.

Tabla 3: Análisis de validez discriminante

\begin{tabular}{|l|c|c|c|c|}
\hline & $F 1$ & $F 2$ & $F 3$ & $F 4$ \\
\hline F1: Aprendizaje de exploración & $\underline{0,820}$ & 0,734 & 0,683 & 0,692 \\
F2: Aprendizaje de explotación & $(0,665 ; 0,803)$ & $\underline{0,824}$ & 0,826 & 0,527 \\
F3: Estrategia de costos & $(0,607 ; 0,759)$ & $(0,773 ; 0,879)$ & $\underline{0,827}$ & 0,521 \\
F4: Estrategia de diferenciación & $(0,616 ; 0,768)$ & $(0,431 ; 0,623)$ & $(0,425 ; 0,617)$ & $\underline{0,796}$ \\
\hline
\end{tabular}


Tabla 4: Análisis de fiabilidad

\begin{tabular}{|l|c|c|c|c|}
\hline \multicolumn{1}{|c|}{ Constructos } & $N^{\circ}$ de ítems & Alfa de Chronbach & IFC & IVE \\
\hline Aprendizaje de exploración & 3 & 0,855 & 0,861 & 0,673 \\
Aprendizaje de explotación & 3 & 0,851 & 0,863 & 0,678 \\
Estrategia de costos & 3 & 0,866 & 0,866 & 0,684 \\
Estrategia de diferenciación & 3 & 0,825 & 0,836 & 0,633 \\
\hline
\end{tabular}

\section{RESULTADOS}

Se realizó un análisis de modelos de regresión múltiple a través del programa SPSS 23 para analizar las hipótesis inicialmente planteadas. En la tabla 5 se muestran los modelos realizados, señalando el nivel de significación de cada uno de los valores estimados $\left({ }^{*} p<0,10 ;{ }^{* *} p<0,05 ;{ }^{* * *} p<0,01\right)$. En primer lugar, no se evidencian problemas de multicolinealidad entre las variables independientes, ya que los factores de inflación de la varianza FIV son inferiores a 10 en todos los modelos (Hair et al., 1999).

Tabla 5: Modelos de regresión

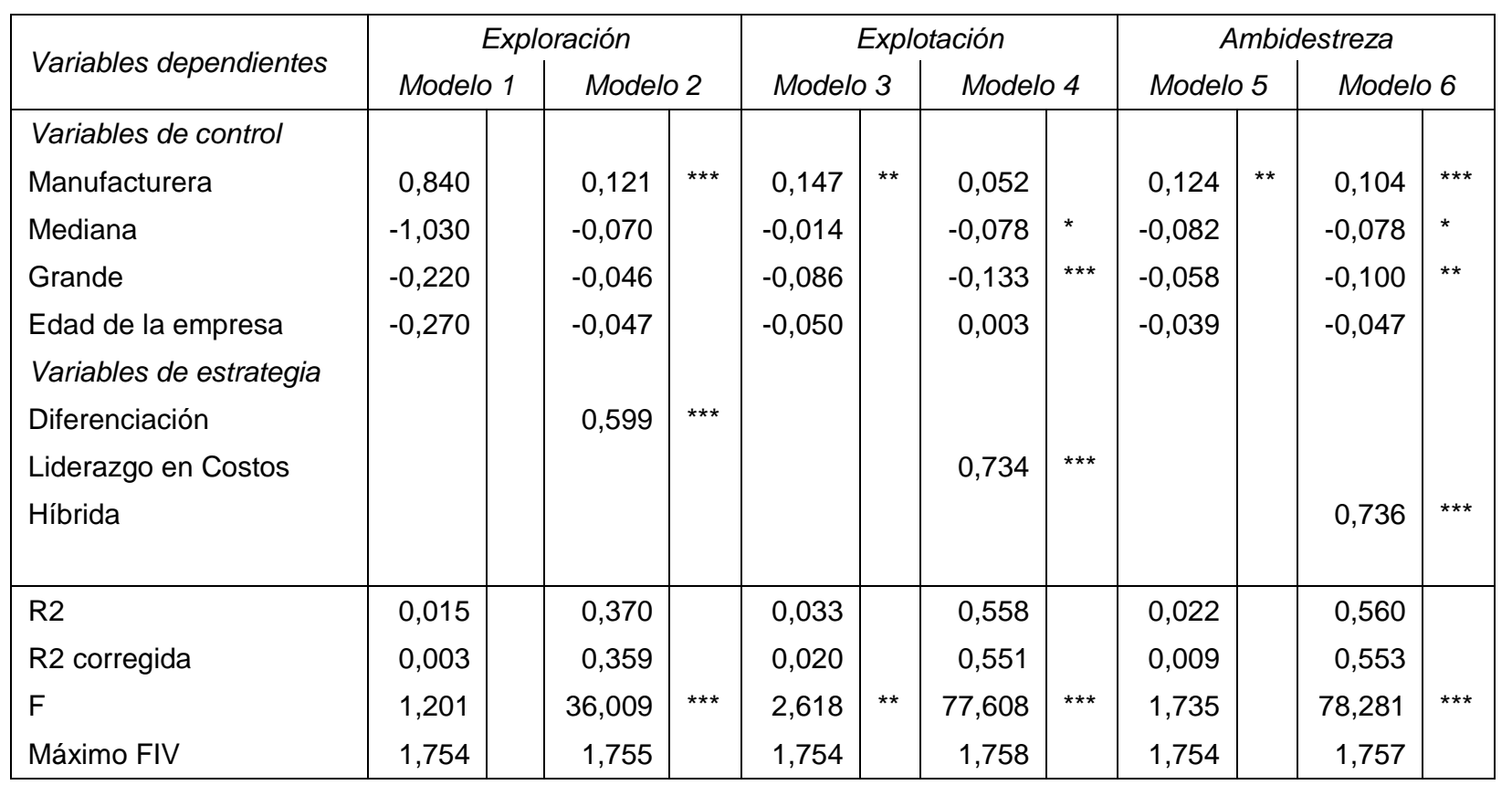

En los modelos 1 y 2, la variable dependiente es la exploración. El modelo 1 utiliza únicamente como regresores las variables de control, de donde se desprende que ninguna de ellas influye en el nivel de exploración (no se presentan coeficientes significativos). El modelo 2 introduce la estrategia de diferenciación, manteniendo las variables de control. En este modelo se observa que la variable introducida es significativa al $99 \%$ y su capacidad predictiva del modelo es elevada (R2 corregido $=0,359$ ), para el tamaño muestral considerado. Con estos resultados se puede confirmar la hipótesis. En el modelo 2 se observa que la pertenencia de la empresa al sector de manufactura también tiene una relación positiva significativa con el aprendizaje de exploración. Para los modelos 3 y 4, se toma como variable dependiente la explotación. En el modelo 3 se hace la regresión considerando sólo las variables de control, identificando que se tiene una leve influencia del sector manufacturero respecto al nivel de explotación de una empresa $(p<0,05)$. El modelo 4 incluye la estrategia de ventaja en costos, que demuestra un coeficiente positivo significativo al $99 \%$, $(p<$ 0,01 ), resultado que permite avalar así la hipótesis 2 . Adicionalmente, se observa que las empresas medianas y grandes tienen un impacto negativo en comparación con las empresas pequeñas (coeficientes negativos), aunque dicha relación es menos significativa en las empresas medianas.

Finalmente, para los modelos 5 y 6 se toma como variable dependiente la ambidestreza. El nivel de ambidestreza es aquel derivado de la ejecución combinada y simultánea de la exploración y la explotación. En el modelo 5 se analizan sólo las variables de control y se observa una influencia positiva de la pertenencia de la empresa al sector manufacturero sobre el nivel de ambidestreza $(p<0,05)$. El modelo 6 introduce la estrategia híbrida como variable independiente y se observa una influencia positiva significativa al $99 \%(p<$ $0,01)$ sobre el nivel de ambidestreza, resultado que permite respaldar la hipótesis 3 . En dicho modelo se observa que el sector de manufactura también tiene una influencia positiva significativa $(p<0,01)$ y que las 
empresas medianas y grandes presentan una influencia negativa en comparación con las empresas pequeñas, aunque su nivel de significación es menor ( $p<0,10$ y $p<0,05$ respectivamente). En este modelo no se incluyen las variables de diferenciación y costos de forma independiente, para evitar la multicolinealidad, ya que la estrategia híbrida es una combinación de las otras dos.

\section{DISCUSIÓN}

Este estudio analizó la estrategia competitiva basada en diferenciación, ventaja en costos e híbrida con respecto a los tipos de aprendizaje de exploración, explotación y ambidestreza, en diversas empresas. En este sentido, se propuso a la explotación (reafirmación y expansión de conocimientos que la empresa ya tiene) y la exploración (consecución de conocimiento nuevos, que la empresa no posee) como elementos de la ambidestreza. Asimismo, se planteó una orientación evaluativa del impacto de la estrategia competitiva de las empresas, sobre la capacidad de generación de conocimiento ambidiestro y sus componentes. Hay conceptos que actualmente vienen tomando fuerza y aplicación en las empresas, como son la innovación, tecnología, investigación, I+D+i, que no son exclusivos de la ciencia, incubadoras de negocios o ángeles inversionistas, estas temáticas hacen una importante contribución al aprendizaje de exploración, porque se convierten en labores propulsoras a cambios y grandes retos empresariales, que facilitan una correspondencia directa con la estrategia de diferenciación, tal y como lo demuestran los resultados del modelo 2. Las empresas que se orienten por la implementación de este tipo de habilidad y estrategia se pueden ubicar en una posición de mayor reputación, continuidad y ventaja competitiva.

El manejo óptimo de los recursos, en los que se incluye además de los materiales, el conocimiento institucionalizado, exige a las empresas mayor nivel de explotación de su experiencia y saber existente, para alcanzar mayores niveles de productividad, eficiencia y competitividad, sin alteración de los costos. Lograr estos fines, deja en evidencia la relación entre el aprendizaje de explotación con la estrategia de ventaja en costos, vínculo que se pudo confirmar en el modelo 4, del apartado de resultados, y que además de contribuir con las buenas prácticas administrativas está agregando valor para todos sus grupos interés. Lo ideal sería que las empresas operaran con una afinidad entre ambas estrategias competitivas y las habilidades de exploración y explotación, para que el resultado de esta combinación, estrategia híbrida y aprendizaje ambidiestro, se extienda a su mayor alcance en el negocio y trascienda los resultados esperados, además de lograr un alto grado de desempeño en la utilización de los bienes, aprovechamiento del capital intelectual, manejo de la imagen corporativa, acentuación del prestigio, aumento de la rentabilidad, lo cual confluye en la permanencia a largo plazo de la empresa.

Frente a estos enfoques, el estudio ha reflejado una correspondencia e impacto positivo, entre la estrategia de diferenciación con el conocimiento de exploración, la estrategia de ventaja en costos con el conocimiento de explotación y la estrategia híbrida con la ambidestreza, permitiendo así corroborar las tres hipótesis planteadas. Comprobación que admite, además, asentir que hay una relación de complementariedad entre el aprendizaje y la estrategia para un mejor desempeño empresarial, porque se logra conseguir el máximo provecho de los recursos tangibles e intangibles. Partiendo del capital humano, se logra la explotación del conocimiento experto, del aprendizaje existente y la experiencia alcanzada, para hacer una buena administración de los bienes materiales y lograr la optimización de los mismos, despertando así un pensamiento creador, arriesgado, motivado al cambio y con el firme propósito de marcar diferencias competitivas que redunde en el éxito empresarial.

En este estudio se pudo identificar que algunas de las variables de control planteadas como el sector o el tamaño, también influyen sobre los tipos de aprendizaje organizacional. Aun así, dicha influencia no se evidenció en cuanto a la edad de la empresa. Puntualmente se observa que la pertenencia de las empresas al sector de manufactura influye en su nivel de aprendizaje de exploración, las empresas pequeñas influyen en el nivel de aprendizaje de explotación y las empresas que presentan ambas características; de manufactura y pequeñas, influyen sobre el aprendizaje ambidiestro de la organización. Se consideran resultados coherentes y acordes con la teoría (Pertusa-Ortega et al., 2009; Baroto et al., 2012; Lapersonne et al., 2015) ya que el concepto que proporciona el balance entre la aplicación simultánea de la exploración y la explotación es el aprendizaje ambidiestro.

Es interesante comparar estos resultados con otros estudios que han relacionado constructos similares. Por ejemplo, Yamakawa et al. (2011), con un panel de empresas norteamericanas, encuentran que las empresas que se centran en la estrategia de liderazgo en costos se beneficiarán más de una mayor proporción de alianzas de explotación, mientras que las empresas con un enfoque en la estrategia de diferenciación se benefician más de una mayor proporción de alianzas de exploración, en su cartera de alianzas. De forma similar, Kim y Huh (2015), con un panel de empresas coreanas de tecnologías de información, concluyen que las empresas que ponen gran énfasis en la estrategia de diferenciación pueden obtener una ventaja 
competitiva sostenible a través de la innovación exploratoria, mientras que aquellas que no lo hacen probablemente tienen dificultades para extender su longevidad a través de actividades de innovación exploratoria y, por lo tanto, pueden beneficiarse más de la innovación explotadora. Por lo tanto, los gerentes deben priorizar sus actividades de innovación de modo que haya un buen ajuste entre sus actividades y la orientación estratégica.

Estudios como estos y gran parte de la literatura predominante en temas de estrategia se han caracterizado por estudiar empresas en contextos de economías desarrolladas con altos niveles de innovación (Norteamérica, Europa occidental, Sudeste asiático). En el caso de esta investigación, se destaca el hecho de encontrar resultados coincidentes con la teoría, pero en el contexto de una economía emergente como Colombia, en la cual las actividades de innovación presentan un menor nivel de madurez y la cultura nacional se caracteriza por una mayor aversión a la incertidumbre y menor orientación al largo plazo (Hofstede et al., 2010). Además de la influencia que estas variables independientes y de control presentan sobre el aprendizaje ambidiestro, podría pensarse que el aprendizaje también depende de la visión que tengan los directivos, de su disposición al cambio y de los retos que contribuyan a una mejor adaptación a su medio ambiente, aseguren continuidad, respondan efectivamente a las amenazas del entorno y mitiguen las adversidades, sin dejar de lado que en esta consecución de objetivos, juegan un papel importante los equipos de trabajo y de colaboradores, que son quienes se alinean a la estrategia empresarial y hacen posible la implementación de tácticas, métodos, procesos, perfeccionan capacidades, explotan su potencial, impulsan mejoras y desarrollan planes decisivos para el éxito empresarial. En futuras investigaciones podrían estudiarse estos factores como antecedentes de las capacidades de aprendizaje.

Dentro de las limitaciones de esta investigación es importante considerar que los resultados se circunscriben al contexto geográfico de la muestra, conformada por empresas colombianas de manufactura y servicios. En este país, el estudio de la ambidestreza organizacional se encuentra en un estado incipiente, lo cual puede ser una oportunidad para que futuras investigaciones profundicen sobre este tema. También sería interesante desarrollar esta investigación en otros tipos de organizaciones como instituciones oficiales e indagar por ejemplo si la naturaleza del sector público se convierte en una restricción o una oportunidad para promover la ambidestreza. Otra limitación de la investigación es su diseño transversal (aplicación del instrumento en un momento de tiempo determinado), sería valioso desarrollar investigaciones longitudinales con las mismas variables, para comprender la evolución dinámica entre la estrategia competitiva y el aprendizaje organizacional. Finalmente, futuras investigaciones podrían analizar la relación entre estos constructos y el desempeño empresarial.

\section{CONCLUSIONES}

De acuerdo a los resultados de esta investigación se respalda el planteamiento de que la estrategia competitiva de una organización está relacionada con los tipos de aprendizaje organizativo que ésta desarrolla. En primer lugar, se encuentra que las organizaciones que siguen una estrategia de diferenciación favorecen el desarrollo del aprendizaje de exploración, ya que las actividades encaminadas a desarrollar atributos únicos para los productos y servicios para que las marcas sean percibidas con un mayor valor por parte del mercado, implica que la empresa desarrolle nuevas competencias y sea capaz de adquirir conocimientos novedosos (exploración) que le permitan reinventarse e innovar de forma permanente. En segundo lugar, las organizaciones que siguen una estrategia de liderazgo en costos favorecen el desarrollo del aprendizaje de explotación, ya que esta estrategia implica el desarrollo de economías de escala y acumulación de experiencia (curvas de aprendizaje) a través de la estandarización de procesos, el mejoramiento continuo y el aprovechamiento de los conocimientos institucionalizados de la organización (explotación), de tal forma que la empresa puede mantener los costos bajos y ampliar su cuota de mercado. Por último, aquellas organizaciones que incorporan ambas estrategias competitivas están desplegando la estrategia híbrida y a su vez favorecen el aprendizaje ambidiestro, puesto que logran desarrollar simultáneamente una oferta de valor superior para los clientes manteniendo una estructura de costos competitiva, lo cual implica la búsqueda permanente de nuevos conocimientos (exploración) y el aprovechamiento óptimo de los conocimientos actuales (explotación). Este último planteamiento desafía la idea tradicional de que las empresas que buscan desarrollar ambas estrategias de forma simultánea quedarían atrapadas a la mitad.

\section{AGRADECIMIENTOS}

Agradecemos el apoyo del Comité para el Desarrollo de la Investigación (CODI) de la Universidad de Antioquia, por su financiación para el desarrollo de esta investigación. 


\section{REFERENCIAS}

Anderson, J.C. y D.W. Gerbing, Structural equation modeling in practice: A review and recommended two-step approach, Psychological Bulletin, 103(3), 411-423 (1988)

Barney, J., Firm resources and the theory of competitive advantage, Journal of Management, 17(1), 99-120 (1991)

Baroto, M.B., M.M.B. Abdullah y H.L. Wan, Hybrid strategy: A new strategy for competitive advantage, International Journal of Business and Management, 7(20), 120-133 (2012)

Baum, J.A.C., S.X. Li y J.M. Usher, Making the next move: How experiential and vicarious learning shape the locations of chains' acquisitions, Administrative Science Quarterly, 45(4), 766-801 (2000)

Boumgarden, P., J. Nickerson y T.R. Zenger, Sailing into the wind: Exploring the relationships among ambidexterity, vacillation, and organizational performance, Strategic Management Journal, 33(6), 587-610 (2012)

Bowman, C., Perceptions of competitive strategy: realised strategy, consensus and performance, Tesis doctoral, Cranfield University, Cranfield, England (1991)

Bowman, C. y D. Faulkner, Competitive and corporate strategy, Irwin, London (1997)

Claver-Cortés, E., E.M. Pertusa-Ortega y J.F. Molina-Azorín, Characteristics of organizational structure relating to hybrid competitive strategy: Implications for performance, Journal of Business Research, 65(7), 993-1002 (2012)

Crossan, M.M., H.W. Lane y R.E. White, An Organizational Learning Framework: From Intuition to Institution, Academy of Management Review, 24(3), 522-537 (1999)

Easterby-Smith, M., M. Crossan y D. Nicolini, Organizational learning: debates past, present and future, Journal of Management Studies, 37(6), 783-796 (2000)

Echeverri, A., N. Lozada y J.E. Arias, Incidencia de las prácticas de gestión del conocimiento sobre la creatividad organizacional, Información Tecnológica, 29(1), 71-82 (2018)

Fornell, C. y D.F. Larcker, Evaluating structural equation models with unobservable variables and measurement error, Journal of Marketing Research, 18(1), 39-50 (1981)

Gupta, A.K., K.G. Smith y C.E. Shalley, The interplay between exploration and exploitation, Academy of Management Journal, 49(4), 693-706 (2006)

Hair, J.F., R.E. Anderson, R.L. Tatham y W.C. Black, Análisis multivariante, 5a Ed., Prentice Hall, Madrid (1999)

He, Z.L. y P.K. Wong, Exploration vs. exploitation: An empirical test of the ambidexterity hypothesis, Organization Science, 15(4), 481-494 (2004)

Hofstede, G., G. J. Hofstede y M. Minkov, Cultures and Organizations: Software of the Mind, $3^{\mathrm{a}}$ Ed., McGraw-Hill, New York (2010)

Junni, P., R.M. Sarala y otros tres autores, Guest editors' introduction: The role of human resources and organizational factors in ambidexterity, Human Resource Management, 54(S1), s1-s28 (2015)

Kim, G. y M. G. Huh, Exploration and organizational longevity: The moderating role of strategy and environment, Asia Pacific Journal of Management, 32(2), 389-414 (2015)

Kim, W.C. y R. Mauborgne, Blue Ocean Strategy: How to Create Uncontested Market Space and Make the Competition Irrelevant, Harvard Business Review Press, Boston (2005)

Lapersonne, A., N. Sanghavi y C. De Mattos, Hybrid Strategy, ambidexterity and environment: toward an integrated typology, Universal Journal of Management, 3(12), 497-508 (2015)

López-Zapata, E., F.E. García-Muiña y S.M. García-Moreno, De la organización que aprende a la organización ambidiestra: evolución teórica del aprendizaje organizativo, Cuadernos de Administración, 25(45), 11-37 (2012)

Lubatkin, M. H., Z. Simsek, Y. Ling y J.F. Veiga, Ambidexterity and performance in small-to medium-sized firms: The pivotal role of top management team behavioral integration, Journal of Management, 32(5), 646-672 (2006)

March, J.G., Exploration and Exploitation in Organizational Learning, Organization Science, 2(1), 71-87 (1991)

Miles, R.E. y C.C. Snow, Organizational strategy, structure, and process, McGraw-Hill, New York (1978)

Navas, J. E. y L.A. Guerras, Fundamentos de dirección estratégica de la empresa, Thomson Reuters, Madrid (2012)

Pertusa-Ortega, E.M., J.F. Molina-Azorín y E. Claver-Cortés, Competitive strategies and firm performance: A comparative analysis of pure, hybrid and 'stuck-in-the-middle'strategies in Spanish firms, British Journal of Management, 20(4), 508$523(2009)$

Porter, M.E., Competitive Strategy, Free Press, New York (1980)

Porter, M.E., The Competitive Advantage: Creating and Sustaining Superior Performance, Free Press, New York (1985)

Raisch, S. y J. Birkinshaw, Organizational ambidexterity: Antecedents, outcomes, and moderators, Journal of Management, 34(3), 375-409 (2008) 
Simanca, M. M., L. A. Montoya y C. A. Bernal, Gestión del conocimiento en cadenas productivas: El caso de la cadena láctea en Colombia, Información Tecnológica, 27(3), 93-106 (2016)

Teece, D.J., Explicating dynamic capabilities: the nature and microfoundations of (sustainable) enterprise performance, Strategic Management Journal, 28(13), 1319-1350 (2007)

Turner, N., J. Swart y H. Maylor, Mechanisms for managing ambidexterity: A review and research agenda, International Journal of Management Reviews, 15(3), 317-332 (2013)

Van Grinsven, M. y M. Visser, Empowerment, knowledge conversion and dimensions of organizational learning, The Learning Organization, 18(5), 378-391 (2011)

Yamakawa, Y., H. Yang y Z.J. Lin, Exploration versus exploitation in alliance portfolio: Performance implications of organizational, strategic, and environmental fit, Research Policy, 40(2), 287-296 (2011)

Yelle, L.E., The learning curve: Historical review and comprehensive survey, Decision Sciences, 10(2), $302-328$ (1979) 